\title{
Picard Successive Approximation Method for Solving Differential Equations Arising in Fractal Heat Transfer with Local Fractional Derivative
}

\author{
Ai-Min Yang, ${ }^{1,2}$ Cheng Zhang, ${ }^{3}$ Hossein Jafari, ${ }^{4}$ Carlo Cattani, ${ }^{5}$ and Ying Jiao ${ }^{6}$ \\ ${ }^{1}$ College of Science, Hebei United University, Tangshan, China \\ ${ }^{2}$ College of Mechanical Engineering, Yanshan University, Qinhuangdao, China \\ ${ }^{3}$ School of Civil Engineering and Architecture, Chongqing Jiaotong University, Chongqing 400074, China \\ ${ }^{4}$ Faculty of Basic Sciences, Department of Mathematics, Ayatollah Amoli Branch, Islamic Azad University, Amol 4615143358, Iran \\ ${ }^{5}$ Department of Mathematics, University of Salerno, Via Ponte don Melillo, Fisciano, 84084 Salerno, Italy \\ ${ }^{6}$ Qinggong College, Hebei United University, Tangshan 063000, China
}

Correspondence should be addressed to Ai-Min Yang; aimin_heut@163.com

Received 12 December 2013; Accepted 1 January 2014; Published 11 February 2014

Academic Editor: Ming Li

Copyright (C) 2014 Ai-Min Yang et al. This is an open access article distributed under the Creative Commons Attribution License, which permits unrestricted use, distribution, and reproduction in any medium, provided the original work is properly cited.

\begin{abstract}
The Fourier law of one-dimensional heat conduction equation in fractal media is investigated in this paper. An approximate solution to one-dimensional local fractional Volterra integral equation of the second kind, which is derived from the transformation of Fourier flux equation in discontinuous media, is considered. The Picard successive approximation method is applied to solve the temperature field based on the given Mittag-Leffler-type Fourier flux distribution in fractal media. The nondifferential approximate solutions are given to show the efficiency of the present method.
\end{abstract}

\section{Introduction}

Engineering problems can be mathematically described by differential equations. Many initial and boundary value problems associated with differential equations can be transformed into problems of solving some approximate integral equations. Heat transfer is described by theory of integral equations. Integral equation arising in heat transfer with smooth condition is valid for continuous media [1-4]. The common methods for solving the equations of heat transfer are purely mathematical are among them; the finite difference techniques (FDT) [5], the regression analysis (RA) [6], the Adomian decomposition method (ADM) [7], the combined Laplace-Adomian method (CLAM) [8], the homotopy analysis method (HAM) [9, 10], the differential transformation method (DTM) [11], the spline-wavelets techniques (SWT) [12], the boundary element method (BEM) [13], the heatbalance integral method (HBIM) $[14,15]$, the variational iteration method (VIM) [16], the local fractional variational iteration method (LFVIM) [17], and the Picard successive approximation method (PSAM) [18].

On the other hand, the nanoscale heat problem can be characterized as fractal behaviors. As usual, the materials are called the Cantor materials. Heat transfer in fractal media with nonsmooth conditions is a hot topic. For example, the heat transfer equations in a medium with fractal geometry [19] and fractal domains [20] were considered. The local fractional transient heat conduction equations based upon the Fourier law within local fractional derivative arising in heat transfer from discontinuous media were presented in [21-24].

Fractional calculus was successfully used to deal with the real world problems [25-30]. There is its limit that the operators do not deal with the local fractional continuous functions (nondifferential functions). Hence, the local fractional Fourier flux [21] is not handled by using some approaches from the classical and fractional operators. This paper focuses on analytical solution to local fractional Fourier flux in fractal 
media by using Picard's successive approximation method [18]. This paper is organized as follows. In Section 2, we give notations to local fractional derivative and integrals and investigate the heat transfer in fractal media. Section 3 is devoted to Picard's successive approximation method based upon local fractional integrals. Analysis solution is shown in Section 4. Conclusions are in Section 5.

\section{Heat Transfer in Fractal Media with Local Fractional Derivative}

In order to study the non-differential solution for the heat problem in fractal media with local fractional derivative, we here begin with the Fourier flux equation in discontinuous media.

The temperature field reads as [21]

$$
T(x, y, z, \tau)=f(x, y, z, \tau) \quad \text { at } \tau>\tau_{0} \text { and in } \Omega,
$$

where $f(x, y, z, \tau)$ is local fractional continuous at fractal domain $\Omega$.

For a given temperature field $T$, a local fractional temperature gradient [21] can be written as follows:

$$
\nabla^{\alpha} T=\frac{\partial^{\alpha} T}{\partial u_{1}^{\alpha}} \vec{e}_{1}^{\alpha}+\frac{\partial^{\alpha} T}{\partial u_{2}^{\alpha}} \vec{e}_{2}^{\alpha}+\frac{\partial^{\alpha} T}{\partial u_{3}^{\alpha}} \vec{e}_{3}^{\alpha},
$$

where the local fractional partial derivative is defined by [2124]

$$
\begin{aligned}
f_{x}^{(\alpha)}\left(x_{0}, y\right) & =\left.\frac{\partial^{\alpha} f(x, y)}{\partial x^{\alpha}}\right|_{x=x_{0}} \\
& =\lim _{x \rightarrow x_{0}} \frac{\Delta^{\alpha}\left(f(x, y)-f\left(x_{0}, y\right)\right)}{\left(x-x_{0}\right)^{\alpha}},
\end{aligned}
$$

where $\Delta^{\alpha}\left(f(x, y)-f\left(x_{0}, y\right)\right) \cong \Gamma(1+\alpha) \Delta\left(f(x, y)-f\left(x_{0}, y\right)\right)$.

Here, the local fractional derivative is defined on the fractal set like a Cantor set. For example, when we consider the Cantor set, we can find the local fractional derivative of discontinuous function $T$ (however, $T$ is a local fractional continuous function).

We consider the heat flux per unit fractal area $\vec{q}$ is proportional to the temperature gradient in fractal medium. Fourier law of heat conduction in fractal medium with local fractional derivative is expressed by [21]

$$
\vec{q}(x, y, z, t)=-K^{2 \alpha} \nabla^{\alpha} T(x, y, z, t),
$$

where $K^{2 \alpha}$ denotes the thermal conductivity of the fractal material, and it is related to fractal dimensions of materials. It is shown that the fractal dimensions of materials are an important characteristic value. Here, we consider the fractal Fourier flow, which is discontinuous; however, it is found that it is local fractional continuous. Like classical Fourier flow, its thermal conductivity is an approximate value for fractal one when $\alpha=1$.

Fourier law of one-dimensional heat conduction equation in fractal media reads as [21]

$$
q(x, t)=-K^{2 \alpha} \frac{d^{\alpha} T(x, t)}{d x^{\alpha}}, \quad \text { at } \tau>\tau_{0} \text { and in } A,
$$

where $K^{2 \alpha}$ denotes the thermal conductivity of the fractal materials.

When $\tau=\tau_{0}$, from (5) we have

$$
q(x)=-K^{2 \alpha} \frac{d^{\alpha} T(x)}{d x^{\alpha}},
$$

at $\tau>\tau_{0}$ and in $A$, where $K^{2 \alpha}$ is the thermal conductivity of the fractal materials. Namely, $T$ is a bi-Lipschitz mapping, and shows the fractal characteristic behavior [21].

Local fractional heat conduction equation with heat generation in fractal media can be written as [21]

$$
K^{2 \alpha} \nabla^{2 \alpha} T+g-\rho_{\alpha} c_{\alpha} \frac{\partial^{\alpha} T}{\partial t^{\alpha}}=0 \quad \text { at } \tau>\tau_{0} \text { and in } \Omega .
$$

Local fractional heat conduction equation with no heat generation in fractal media is suggested as $[21,22]$

$$
K^{2 \alpha} \nabla^{2 \alpha} T-\rho_{\alpha} c_{\alpha} \frac{\partial^{\alpha} T}{\partial t^{\alpha}}=0 \quad \text { at } \tau>\tau_{0} \quad \text { and in } \Omega \text {, }
$$

where $\nabla^{2 \alpha}$ is a local fractional Laplace operator [21].

\section{The Method}

In this section, we discuss the Picard successive approximation method. Meanwhile, we transfer the Fourier law of onedimensional heat conduction equation in fractal media into the local fractional Volterra integral equation of the second kind.

3.1. Picard's Successive Approximation Method. This method is first proposed in [18]. Here we will give a short introduction to Picard's successive approximation method within the local fractional calculus.

In this method, we set

$$
u_{0}(x)=f(x)
$$

We give the first approximation $u_{1}(x)$ by

$$
u_{1}(x)=f(x)+\frac{\lambda^{\alpha}}{\Gamma(1+\alpha)} \int_{0}^{x} K(x, t) u_{0}(x)(d t)^{\alpha},
$$

where the local fractional integral of $f(x)$ of order $\alpha$ in the interval $[a, b]$ is defined as follows [21-24]:

$$
\begin{aligned}
a_{a}^{(\alpha)} f(x) & =\frac{1}{\Gamma(1+\alpha)} \int_{a}^{b} f(t)(d t)^{\alpha} \\
& =\frac{1}{\Gamma(1+\alpha)} \lim _{\Delta t \rightarrow 0} \sum_{j=0}^{j=N-1} f\left(t_{j}\right)\left(\Delta t_{j}\right)^{\alpha}
\end{aligned}
$$

with $\Delta t_{j}=t_{j+1}-t_{j}, \Delta t=\max \left\{\Delta t_{0}, \Delta t_{1}, \Delta t_{2}, \ldots\right\}$, and $\left[t_{j}, t_{j+1}\right]$, $j=0, \ldots, N-1, t_{0}=a$, and $t_{N}=b$.

Here, we find that the equality $u_{1}(x)$ is a local fractional continuous function if $f(x), K(x, t)$ and $u_{0}(x)$ are local fractional continuous functions. 
Continuing in this manner, we have the infinite sequences of functions

$$
u_{0}(x), u_{1}(x), u_{2}(x), \ldots, u_{n}(x), \ldots
$$

such that the recurrence equations are given by

$$
\begin{array}{r}
u_{n}(x)=f(x)-\frac{1}{\Gamma(1+\alpha)} \int_{0}^{x} K(x, t) u_{n-1}(x)(d t)^{\alpha} \\
(n=1,2,3, \ldots)
\end{array}
$$

where $u_{0}(x)$ is equivalent to any selected function, which is the local fractional continuous function.

Hence, we have successive approximation as follows:

$$
\begin{gathered}
u_{1}(x)=f(x)+\frac{\lambda^{\alpha}}{\Gamma(1+\alpha)} \int_{0}^{x} K(x, t) f(t)(d t)^{\alpha}, \\
\vdots \\
u_{n}(x)=f(x)+\frac{\lambda^{\alpha}}{\Gamma(1+\alpha)} \int_{0}^{x} K(x, t) u_{n-1}(x)(d t)^{\alpha} .
\end{gathered}
$$

Thus, at the limit, the solution $u(x)$ is written as

$$
u(x)=\lim _{n \rightarrow \infty} u_{n}(x) .
$$

3.2. An Alternative Method from Local Fractional Derivative to Local Fractional Volterra Integral Equations. We directly observe that the local fractional differential equation of $\alpha$ order

$$
K^{2 \alpha} \frac{d^{\alpha} T}{d x^{\alpha}}=q(x) \quad(0 \leq x \leq b)
$$

can be written immediately as the local fractional Volterra integral equation of thee second kind in the form

$$
T(x)=T(a)+\frac{\lambda^{\alpha}}{\Gamma(1+\alpha)} \int_{0}^{x} q(t)(d t)^{\alpha},
$$

where $\lambda=1 / K^{2}$.

The Mittag-Leffler type Fourier flux distribution in fractal media can be written as follows:

$$
q(x)=q_{t}(t, x)=E_{\alpha}(x-t)^{\alpha} T(t) \quad(0 \leq t \leq b) .
$$

Making use of (18), we can get the local fractional Volterra integral equation of the second kind in the form:

$$
T(x)=T(a)+\frac{\lambda^{\alpha}}{\Gamma(1+\alpha)} \int_{0}^{x} E_{\alpha}(x-t)^{\alpha} T(t)(d t)^{\alpha} .
$$

\section{Approximate Solutions for Local Fractional Volterra Integral Equation of the Second Kind}

Let us assume that the zeroth approximation is

$$
u_{0}(x)=0
$$

Then the first approximation can be written as follows:

$$
u_{1}(x)=f(x)=T(a) .
$$

Here we obtain the second approximation, which reads as

$$
u_{2}(x)=f(x)+\frac{\lambda^{\alpha}}{\Gamma(1+\alpha)} \int_{0}^{x} E_{\alpha}(x-t)^{\alpha} u_{1}(t)(d t)^{\alpha} .
$$

Proceeding in this manner, we have the third approximation in the following form:

$$
u_{3}(x)=f(x)+\frac{\lambda^{\alpha}}{\Gamma(1+\alpha)} \int_{0}^{x} E_{\alpha}(x-t)^{\alpha} u_{2}(t)(d t)^{\alpha} .
$$

Hence, continuing in this manner, we obtain

$$
\begin{aligned}
u_{n}(x)= & f(x)+\frac{\lambda^{\alpha}}{\Gamma(1+\alpha)} \int_{0}^{x} E_{\alpha}(x-t)^{\alpha} u_{n-1}(t)(d t)^{\alpha} \\
= & f(x)+\frac{\lambda^{\alpha}}{\Gamma(1+\alpha)} \\
& \times \int_{0}^{x}\left(1+\cdots+\frac{\lambda^{(n-1) \alpha}(x-t)^{(n-1) \alpha}}{\Gamma(1+(n-1) \alpha)}\right) \\
& \times E_{\alpha}(x-t)^{\alpha} f(t)(d t)^{\alpha} .
\end{aligned}
$$

Taking the limit, we have

$$
\begin{aligned}
u(x)= & \lim _{n \rightarrow \infty} u_{n}(x) \\
= & T(a)+\frac{\lambda^{\alpha}}{\Gamma(1+\alpha)} \\
& \times \int_{0}^{x} E_{\alpha}\left[(x-t)^{\alpha}\left(1+\lambda^{\alpha}\right)\right] T(a)(d t)^{\alpha} \\
= & T(a)\left\{\frac{2 \lambda^{\alpha}}{1+\lambda^{\alpha}} E_{\alpha}\left[x^{\alpha}\left(1+\lambda^{\alpha}\right)\right]\right\} \\
= & T(a)\left\{\frac{2}{K^{2 \alpha}+1} E_{\alpha}\left[x^{\alpha}\left(1+\frac{1}{K^{2 \alpha}}\right)\right]\right\},
\end{aligned}
$$

where the term $E_{\alpha}\left[x^{\alpha}\left(1+\lambda^{\alpha}\right)\right]$ is a Mittag-Leffler type Fourier flux distribution in fractal media, which is related to the fractal coarse-grained mass function $[21,24]$. When $K=1$, we get $u(x)=T(a) E_{\alpha}\left[2 x^{\alpha}\right]$. The nondifferentiable solution of (25) for parameters $K=1, \alpha=\ln 2 / \ln 3$, and $T(a)=1$ is shown in Figure 1; the non-differentiable solution of (25) for parameters $K=1, \alpha=\ln 2 / \ln 3$, and $T(a)=2$ is shown in Figure 2; the non-differentiable solution of (25) for parameters $K=1, \alpha=\ln 2 / \ln 3$, and $T(a)=3$ is shown in Figure 3; the non-differentiable solution of (25) for parameters $K=1, \alpha=\ln 2 / \ln 3$, and $T(a)=4$ is shown in Figure 4.

\section{Conclusions}

This work studied the Fourier law of one-dimensional heat conduction equation in fractal media. Mittag-Leffler type 


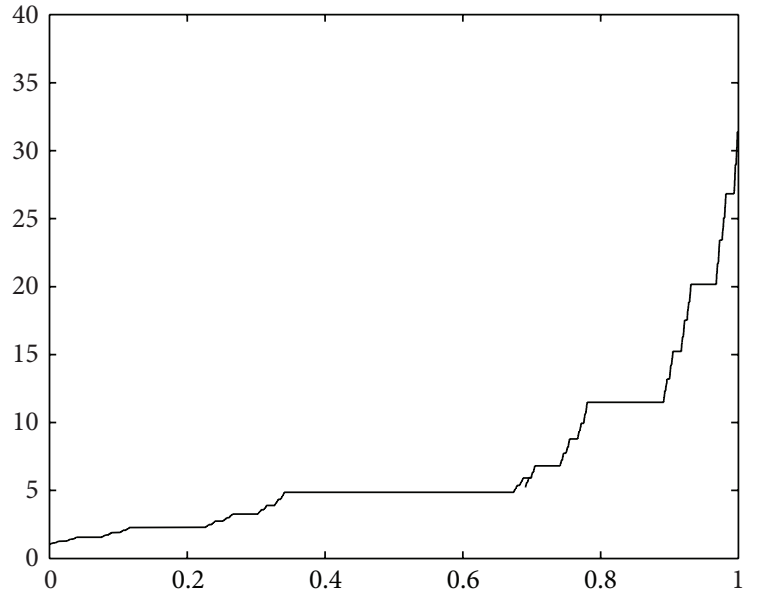

Figure 1: The nondifferentiable solution for Mittag-Leffler type Fourier flux distribution for parameters $K=1, \alpha=\ln 2 / \ln 3$, and $T(a)=1$.

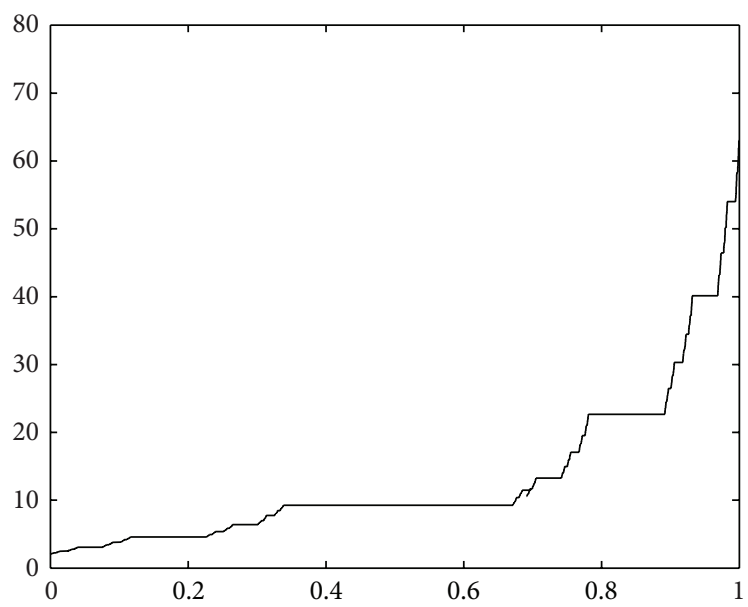

Figure 2: The nondifferentiable solution for Mittag-Leffler type Fourier flux distribution for parameters $K=1, \alpha=\ln 2 / \ln 3$, and $T(a)=2$.

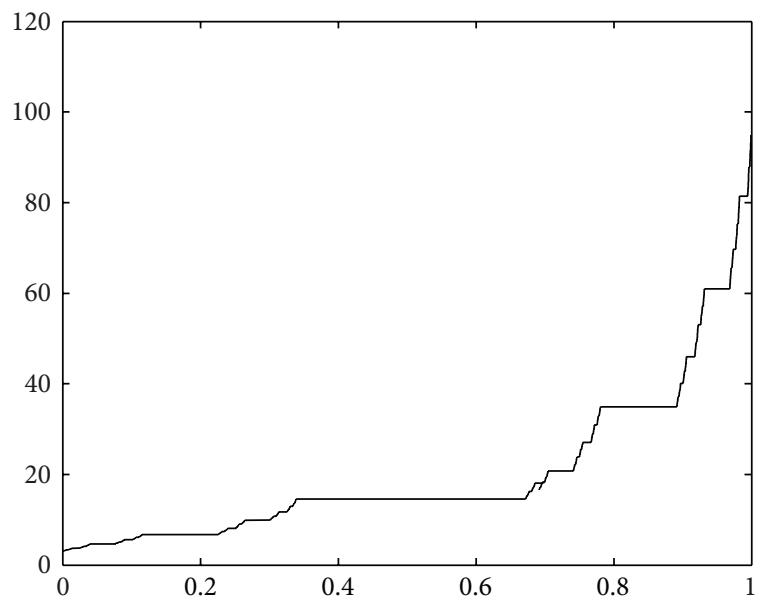

FIgURE 3: The nondifferentiable solution for Mittag-Leffler type Fourier flux distribution for parameters $K=1, \alpha=\ln 2 / \ln 3$, and $T(a)=3$.

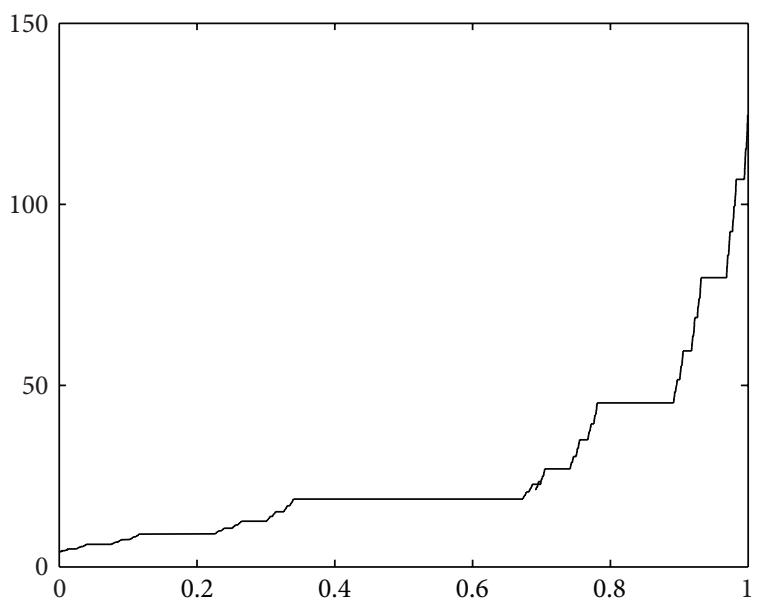

Figure 4: The nondifferentiable solution for Mittag-Leffler type Fourier flux distribution for parameters $K=1, \alpha=\ln 2 / \ln 3$, and $T(a)=4$.

Fourier flux distribution in fractal media with temperature field effect was considered. An approximation solution for the local fractional Volterra integral equation of the second kind derived from Fourier law of one-dimensional heat conduction equation for heat conduction in discontinuous media was studied by using Picard's successive approximation method. The non-differential approximate solutions were given to show the efficiency of the present method.

\section{Conflict of Interests}

The authors declare that there is no conflict of interests regarding the publication of this paper.

\section{Acknowledgments}

This work was supported by National Scientific and Technological Support Projects (no. 2012BAE09B00), the National Natural Science Foundation of China (no. 51274270), and the National Natural Science Foundation of Hebei Province (no. E2013209215).

\section{References}

[1] J. Crank and P. Nicolson, "A practical method for numerical evaluation of solutions of partial differential equations of the heat-conduction type," Mathematical Proceedings of the Cambridge Philosophical Society, vol. 43, pp. 50-67, 1947.

[2] T. M. Shih, "A literature survey on numerical heat transfer," Numerical Heat Transfer, vol. 5, no. 4, pp. 369-420, 1982.

[3] B. C. Choi and S. W. Churchill, "A technique for obtaining approximate solutions for a class of integral equations arising in radiative heat transfer," International Journal of Heat and Fluid Flow, vol. 6, no. 1, pp. 42-48, 1985.

[4] Y. Z. Povstenko, "Fractional heat conduction equation and associated thermal stress," Journal of Thermal Stresses, vol. 28, no. 1, pp. 83-102, 2004. 
[5] M. Dehghan, "The one-dimensional heat equation subject to a boundary integral specification," Chaos, Solitons \& Fractals, vol. 32, no. 2, pp. 661-675, 2007.

[6] Y. Ioannou, M. M. Fyrillas, and C. Doumanidis, "Approximate solution to Fredholm integral equations using linear regression and applications to heat and mass transfer," Engineering Analysis with Boundary Elements, vol. 36, no. 8, pp. 1278-1283, 2012.

[7] S. Nadeem and N. S. Akbar, "Effects of heat transfer on the peristaltic transport of MHD Newtonian fluid with variable viscosity: application of adomian decomposition method," Communications in Nonlinear Science and Numerical Simulation, vol. 14, no. 11, pp. 3844-3855, 2009.

[8] A.-M. Wazwaz and M. S. Mehanna, "The combined LaplaceAdomian method for handling singular integral equation of heat transfer," International Journal of Nonlinear Science, vol. 10, no. 2, pp. 248-252, 2010.

[9] S. Abbasbandy, "The application of homotopy analysis method to nonlinear equations arising in heat transfer," Physics Letters A, vol. 360, no. 1, pp. 109-113, 2006.

[10] B. Raftari and K. Vajravelu, "Homotopy analysis method for MHD viscoelastic fluid flow and heat transfer in a channel with a stretching wall," Communications in Nonlinear Science and Numerical Simulation, vol. 17, no. 11, pp. 4149-4162, 2012.

[11] A. A. Joneidi, D. D. Ganji, and M. Babaelahi, "Differential transformation method to determine fin efficiency of convective straight fins with temperature dependent thermal conductivity," International Communications in Heat and Mass Transfer, vol. 36, no. 7, pp. 757-762, 2009.

[12] C. Cattani and E. Laserra, "Spline-wavelets techniques for heat propagation," Journal of Information \& Optimization Sciences, vol. 24, no. 3, pp. 485-496, 2003.

[13] N. Simões, A. Tadeu, J. António, and W. Mansur, "Transient heat conduction under nonzero initial conditions: a solution using the boundary element method in the frequency domain," Engineering Analysis with Boundary Elements, vol. 36, no. 4, pp. 562-567, 2012.

[14] J. Hristov, "Approximate solutions to fractional sub-diffusion equations: the heat-balance integral method," The European Physical Journal, vol. 193, no. 1, pp. 229-243, 2011.

[15] J. Hristov, "Heat-balance integral to fractional (half-time) heat diffusion sub-model," Thermal Science, vol. 14, no. 2, pp. 291-316, 2010.

[16] D. D. Ganji and H. Sajjadi, "New analytical solution for natural convection of Darcian fluid in porous media prescribed surface heat flux," Thermal Science, vol. 15, no. 2, pp. 221-227, 2011.

[17] X. J. Yang and D. Baleanu, "Fractal heat conduction problem solved by local fractional variation iteration method," Thermal Science, vol. 17, no. 2, pp. 625-628, 2013.

[18] X.-J. Yang, "Picard's approximation method for solving a class of local fractional Volterra integral equations," Advances in Intelligent Transportation Systems, vol. 1, no. 3, pp. 67-70, 2012.

[19] R. R. Nigmatullin, "The realization of the generalized transfer equation in a medium with fractal geometry," Physica Status Solidi B, vol. 133, no. 1, pp. 425-430, 1986.

[20] K. Davey and R. Prosser, "Analytical solutions for heat transfer on fractal and pre-fractal domains," Applied Mathematical Modelling, vol. 37, no. 1-2, pp. 554-569, 2013.

[21] X. J. Yang, Advanced Local Fractional Calculus and Its Applications, World Science, New York, NY, USA, 2012.

[22] Y. Z. Zhang, A. M. Yang, and X.-J. Yang, "1-D heat conduction in a fractal medium: a solution by the local fractional Fourier series method," Thermal Science, vol. 17, no. 3, pp. 953-956, 2013.
[23] M.-S. Hu, D. Baleanu, and X.-J. Yang, "One-phase problems for discontinuous heat transfer in fractal media," Mathematical Problems in Engineering, vol. 2013, Article ID 358473, 3 pages, 2013.

[24] X. J. Yang, Local Fractional Functional Analysis and Its Applications, Asian Academic, Hong Kong, 2011.

[25] M. Li and W. Zhao, "On bandlimitedness and lag-limitedness of fractional Gaussian noise," Physica A, vol. 392, no. 9, pp. 19551961, 2013.

[26] M. Li, "Approximating ideal filters by systems of fractional order," Computational and Mathematical Methods in Medicine, vol. 2012, Article ID 365054, 6 pages, 2012.

[27] M. Li and W. Zhao, "On $1 / f$ noise," Mathematical Problems in Engineering, vol. 2012, Article ID 673648, 23 pages, 2012.

[28] M. H. Heydari, M. R. Hooshmandasl, F. M. Maalek Ghaini, and M. Li, "Chebyshev wavelets method for solution of nonlinear fractional integro-differential equations in a large interval," Advances in Mathematical Physics, vol. 2013, Article ID 482083, 12 pages, 2013.

[29] S. S. Ray and R. K. Bera, "Analytical solution of a fractional diffusion equation by Adomian decomposition method," Applied Mathematics and Computation, vol. 174, no. 1, pp. 329-336, 2006.

[30] Q. Wang, "Numerical solutions for fractional KdV-Burgers equation by Adomian decomposition method," Applied Mathematics and Computation, vol. 182, no. 2, pp. 1048-1055, 2006. 


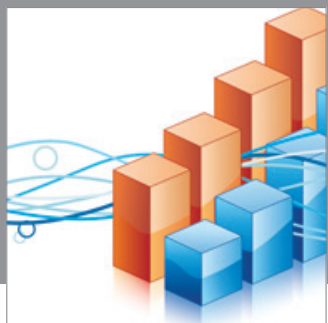

Advances in

Operations Research

mansans

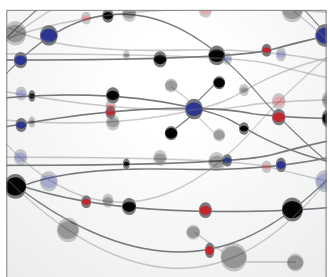

The Scientific World Journal
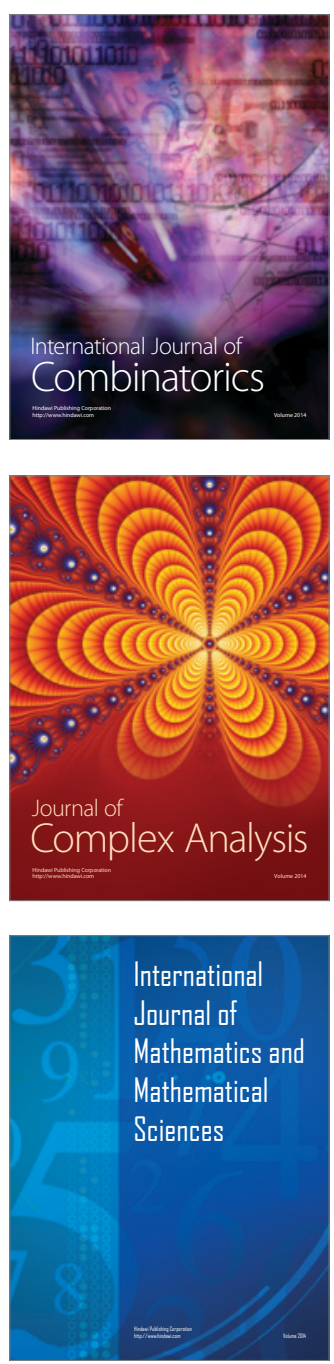
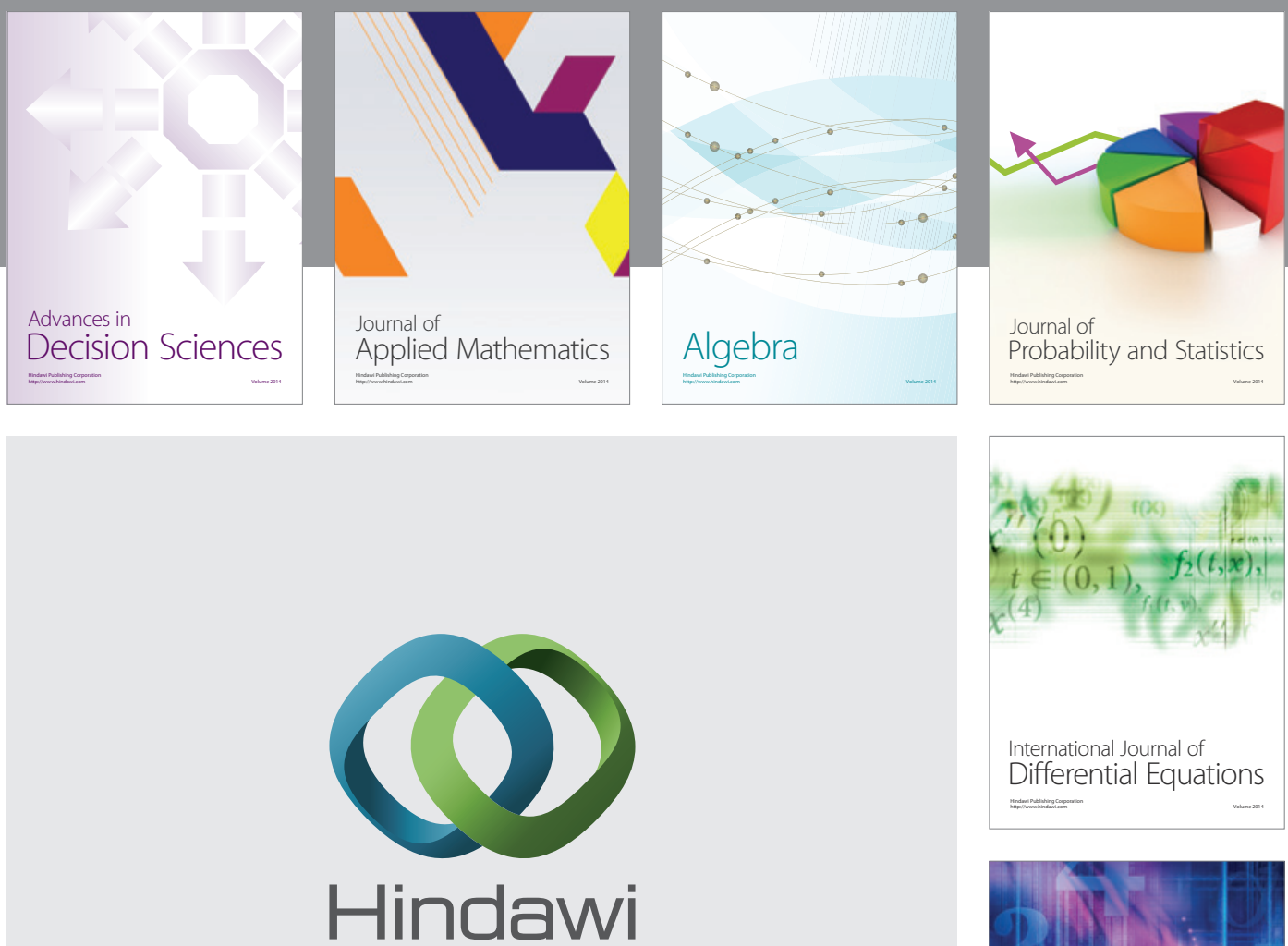

Submit your manuscripts at http://www.hindawi.com
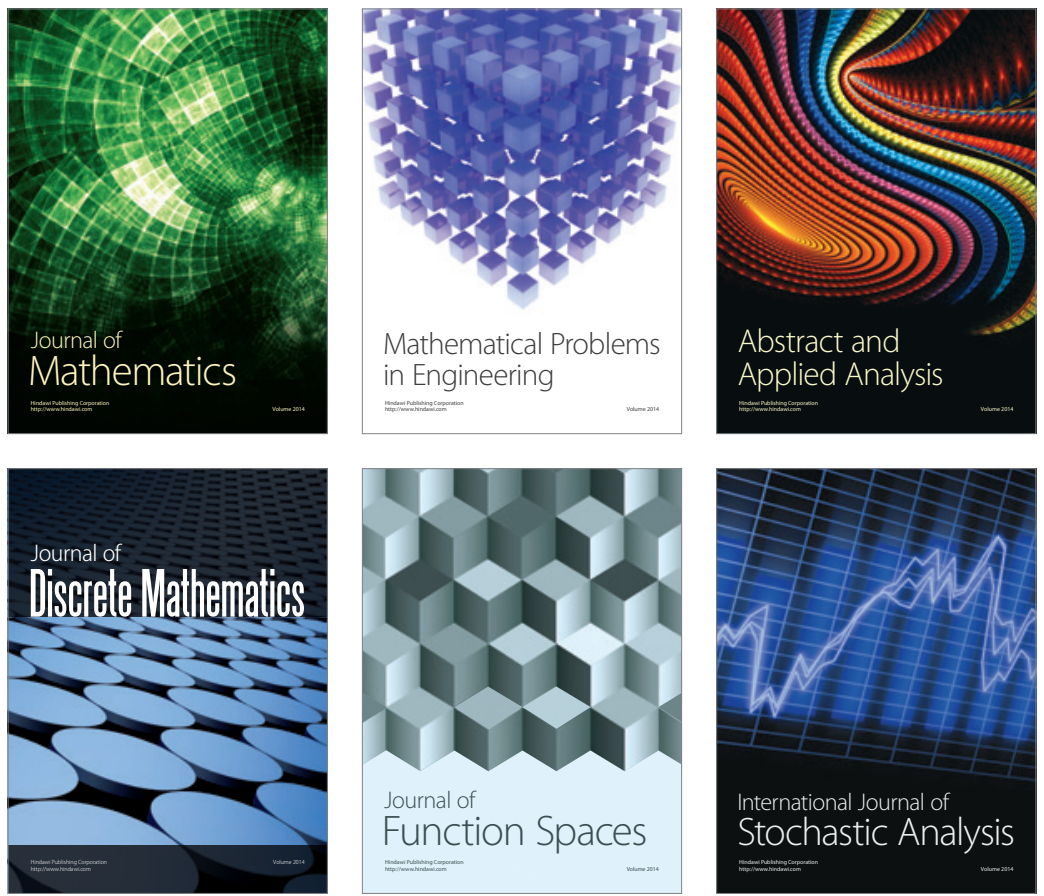

Journal of

Function Spaces

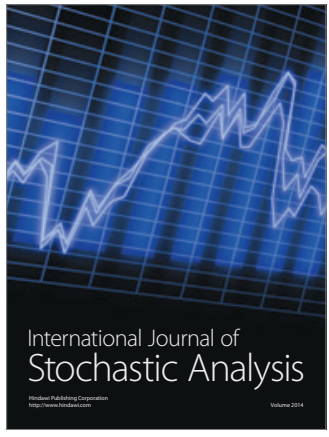

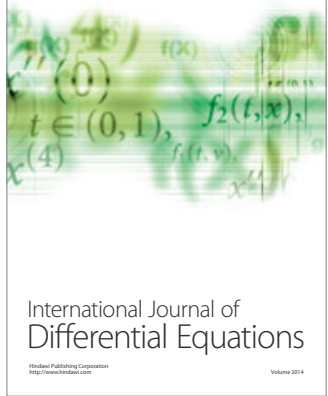
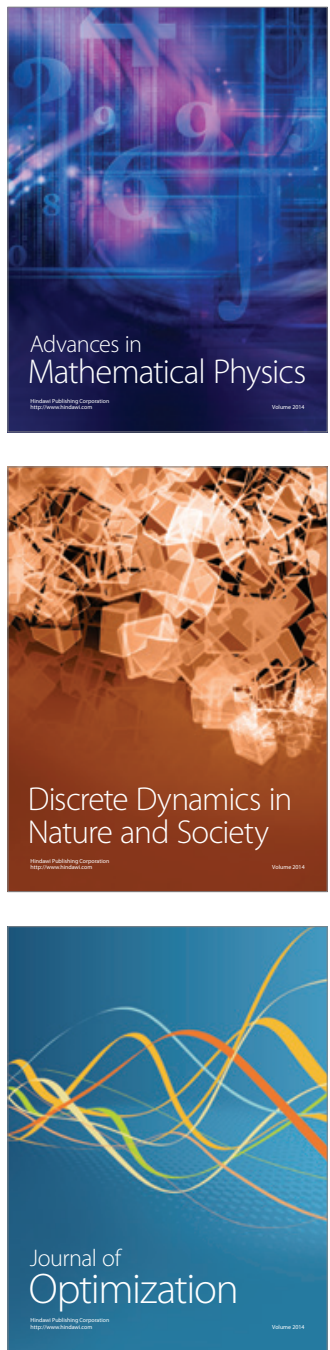